\title{
Enfoque multivariado en experimentos de extracción de madera en plantaciones forestales
}

\section{Multivariate approach in timber harvesting experiments in forest plantations}

\author{
Ouorou Ganni Mariel Gueral*, José Antônio Aleixo da Silva', Rinaldo Luiz Caraciolo Ferreira', \\ Daniel Alberto Álvarez Lazo², Héctor Barrero Medel² y Andrés Loreto Díaz Pita
}

\begin{abstract}
1 Universidade Federal Rural de Pernambuco Departamento de Ciência Florestal. Laboratorio de Biometria e Manejo Florestal. Recife, Pernambuco, Brasil. jaaleixo@gmail.com; rinaldo.ferreira@ufrpe.br

2 Universidad de Pinar del Río "Hermanos Saíz Montes de Oca". Departamento Forestal. Pinar del Río, Cuba. daniel@upr.edu.cu; hbarrero@upr.edu.cu
\end{abstract}

\author{
3 Universidad de Pinar del Río "Hermanos Saíz Montes \\ de Oca". Departamento Agropecuario. Pinar del Río,
} Cuba.adiaz67@upr.edu.cu

\section{RESUMEN}

El presente trabajo se realizó con el objetivo de evaluar, técnica y económicamente la extracción de madera en plantaciones de Pinus caribaea var. caribaea de la empresa forestal Macurije, ubicada en la provincia de Pinar del Río, Cuba. Para ello, se llevaron a cabo estudios de tiempos y movimientos teniendo como criterios de evaluación "productividad" y "costo unitario" de extracción de la madera. La evaluación del desempeño de los tractores articulados (skidders) y de las yuntas de bueyes en diferentes sistemas de aprovechamiento y clases de pendiente se realizó por medio de experimentos factoriales $(3 \times 3 \times 2)$ univariados y multivariados (costo unitario y/o productividad), siguiendo un diseño al azar en bloques $\left(\mathrm{B}_{1}=21\right.$ años y $\mathrm{B}_{2}=32$ años), con los factores "maquinaria" y "pendiente" con tres niveles y "sistema de aprovechamiento" con dos niveles. Se evidenciaron la viabilidad y la utilidad del enfoque multivariado en la evaluación de la extracción de madera; es más viable extraer la madera con el sistema de tronco entero en plantaciones de 32 años. La interacción maquinaria-pendiente tuvo influencia significativa en la variación del conjunto costo-productividad. El skidder John Deere 548E, por presentar menor costo unitario y mayor productividad que el J-80A, es el recomendado para la extracción semimecanizada de la madera. El más viable es la yunta de bueyes por su mayor rentabilidad. Sin embargo, por su baja productividad, solo podrá ser utilizado en combinación con otras máquinas o en periodos de producción desacelerada. Su uso exclusivo no es práctico ya que implicaría aumentar significativamente su cantidad para compensar su baja productividad.

PALABRAS CLAVE: aprovechamiento forestal, Cuba, costo unitario, arreglo factorial multivariado, productividad.

\section{ABSTRACT}

This work was carried out with the objective of evaluating, technically and economically, timber extraction in plantations of Pinus caribaea var. caribaea of Macurije forest company located in the province of Pinar del Río, Cuba. For this, time and movement studies were carried out using "productivity" and "unit cost" of wood extraction as evaluation criteria. The skidders and oxen performance evaluation, in different harvest systems and slope classes, was performed through univariate and multivariate (cost and/or productivity) factorial experiments $(3 \times 3 \times 2)$, following a randomized block design $\left(\mathrm{B}_{1}=21\right.$ years and $\mathrm{B}_{2}=32$ years), with the factors "machinery" and "slope" with three and "harvest systems" with two levels. The feasibility and usefulness of the multivariate approach in the evaluation of timber extraction were evidenced; timber extraction is more viable with the tree length harvest systems in stands of 32 years. The interaction machinery-slope had a significant influence on the variation of the whole cost-productivity. The John Deere $548 \mathrm{E}$ skidder, for presenting lower unit cost and higher productivity than the $\mathrm{J}-80 \mathrm{~A}$, is recommended for semi-mechanized timber extraction. The most viable is the yoke of oxen because of its greater profitability. However, due to its low productivity, it can only be used in combination with other machines or during periods of decelerated production. Its exclusive use is impracticable since it would imply significantly increasing its quantity to compensate for its low productivity.

KEYWORDS: forest harvesting, Cuba, unit cost, multivariate factorial arrangement, productivity. 


\section{INTRODUCCIÓN}

El aprovechamiento forestal es la actividad más costosa del proceso productivo forestal (Santos, Fernades, Silva, Teixeira y Souza, 2016). En esta actividad, la extracción y el transporte de la madera representan más de $50 \%$ de los costos de la madera puesta en la industria (Cándano, 2003; Machado, 2014). Esta alta participación en los costos de producción lleva a las empresas forestales a buscar continuamente alternativas para minimizar los costos de extracción y transporte de madera. Las empresas forestales cubanas no son una excepción y lo anterior refleja de forma fidedigna sus condiciones productivas, principalmente las de la empresa forestal Macurije.

Las presiones adicionales traídas por el aumento constante de la demanda en productos madereros y no madereros llevaron al Ministerio de Agricultura de Cuba (Minagri) a extender, a las empresas forestales cubanas, el proceso de perfeccionamiento empresarial que incluye entre sus principales metas, reducir costos e incrementar rendimientos y producción. El alcance de esas metas tiene que pasar necesariamente por un incremento en la introducción de máquinas, herramientas, piezas de repuestos importadas, así como recursos monetarios y mano de obra (Cándano, 2003). Con la importación de nuevas tecnologías en el país y su introducción en las empresas, estudios de desempeño local de estas fueron necesarios en toda la isla para el conocimiento de sus características específicas. Estos estudios específicos son fundamentales para la selección de los equipos necesarios para las actividades de mecanización (Schettino, Minette y Souza, 2015) y sirven de apoyo para la toma de decisión operacional (Hiesl y Benjamin, 2013).

Los tractores articulados (skidders) son las máquinas más utilizadas en la extracción de madera y se definen como tractores forestales articulados que realizan la extracción de madera, arrastrando las trozas desde el área de corte hasta el acopiadero superior, cuyo material rodante puede ser de neumáticos, semiesteras o esteras (Lopes, Missel, Dias y Fiedler, 2007). La movilidad, aspecto que define la productividad y costo de los skidders, depende de su desempeño en la tracción de la madera, el cual es influenciado por las condiciones del terreno, principalmente la pendiente (Đuka, Pentek, Horvat y Poršinsky, 2016). En la actualidad, el aprovechamiento forestal cuenta con equipos altamente productivos y multifuncionales pero cada vez más pesados (Szymczak et al., 2014). El intenso tráfico de esos equipos dentro de los rodales forestales ha causado problemas ambientales, principalmente la compactación de los suelos (Sampietro y Lopes, 2011). La recurrencia de estos problemas provocó la reintroducción de la tracción animal en las actividades de aprovechamiento forestal, incluso en países altamente industrializados, principalmente en la explotación de los bosques nativos para mitigar daños que pueden comprometer el futuro desarrollo de esos bosques. Además de las razones ambientales, la tracción animal también se usa en países menos industrializados por otras razones, como la falta de red de caminos forestales, la falta de equipos mecanizados, terrenos inclinados que no permiten la extracción mecanizada o semimecanizada, pequeñas áreas de cosecha y bajo volumen de cosecha (Ezzati, Najafi y Durston, 2011). Los principales animales utilizados son: bueyes, burros, mulas, caballos, elefantes, llamas y yaks (Dykstra y Heinrich, 1996). En Cuba, específicamente en la empresa Macurije, los animales utilizados son los bueyes por sus bajos costos operacionales y la mejora de rendimiento proporcionada por su combinación con los skidders (Cándano, 1998).

La realización de los últimos estudios sobre la extracción de madera en la empresa forestal Macurije, objeto del presente estudio, se remonta a los realizados por Cándano (1998). En 20 años, se registraron muchos cambios en los escenarios productivos preexistentes en la empresa, principalmente en la maquinaria de extracción y transporte de madera y en la experiencia laboral de los operadores. Ejemplo de ello es la reciente introducción de los skidders chinos J-80A que se encuentran en fase de prueba y para los cuales no se realizó ningún estudio de desempeño local. Debido a la cantidad de factores que afectan la extracción de madera, es necesario realizar estudios de las características técnico-económicas de la maquinaria de extracción en las 
condiciones en las cuales serán utilizadas (Pereira, Lopes y Dias, 2015). Entre esos factores se pueden citar las dimensiones de las trozas (Machado y Lopes, 2000), la pendiente y el tipo de maquinaria (Sant'anna, 1992).

La fuerte asociación entre los factores supracitados y su significativa influencia en el desempeño de las máquinas han llevado al uso de arreglos factoriales para analizar las posibles interacciones existentes entre estos. Sin embargo, los enfoques han sido mayormente univariados considerando de forma aislada, como principales criterios de evaluación, las variables "costo unitario" o "productividad", entre otras. Esa práctica (uso aislado de los referidos criterios de evaluación) suele generar resultados divergentes que dificultan un análisis conclusivo que permita la selección de la maquinaria adecuada y el sistema de aprovechamiento adecuado en determinado escenario (clase de pendiente) para obtener mayor productividad y menor costo unitario.

Con base en lo anterior y suponiendo que una evaluación basada simultáneamente en el costo unitario y en la productividad proporcionaría resultados más equilibrados para toma de decisiones menos subjetivas, el presente estudio se realizó con el siguiente objetivo.

\section{OBJETIVOS}

Evaluar técnica y económicamente, basándose simultáneamente en los criterios "costo unitario" y "productividad", el desempeño de los equipos de extracción de madera en plantaciones de Pinus caribaea Morelet var. caribaea Barr. \& Golf. en diferentes escenarios en la empresa forestal Macurije, Pinar del Río, Cuba.

\section{MATERIALES Y MÉTODOS}

Caracterización del área de estudio y de las máquinas y equipos de extracción de madera

Esta investigación se realizó en áreas montañosas de plantaciones de P. caribaea var. caribaea de la Unidad Básica de Producción Forestal (UBPF) "las cañas" perteneciente a la unidad silvícola (US) Mantua de la Empresa Forestal Macurije, ubicada aproximadamente entre las coordenadas geográficas $22^{\circ} 06^{\prime}$ a $22^{\circ} 42^{\prime}$ latitud Norte y $83^{\circ} 48^{\prime}$ a $84^{\circ} 23^{\prime}$ longitud oeste en la región más occidental de la provincia de Pinar del Río, Cuba. Las principales características de los rodales en los que se realizaron los experimentos se resumen en la tabla 1.

TABLA 1. Características de los rodales de P. caribaea var. caribaea utilizados para los experimentos

\begin{tabular}{cccccccc}
\hline Número del lote & \multicolumn{3}{c}{80} & & 81 & \multicolumn{3}{c}{88} \\
\hline Número del rodal & 18 & 20 & 06 & 09 & 12 & 18 \\
\hline Áreas (ha) & 7.67 & 6.00 & 28.7 & 27.2 & 19.8 & 16.4 \\
Pendiente media (\%) & 05 & 04 & 17 & 12 & 11 & 24 \\
Edad (años) & 21.00 & 32.00 & 32.00 & 21.00 & 32.00 & 21.00 \\
DAPm (cm) & 20.83 & 21.80 & 22.10 & 20.10 & 22.40 & 20.95 \\
$\mathrm{H}_{\mathrm{m}}(\mathrm{m})$ & 15.5 & 16.00 & 17.21 & 15.70 & 16.00 & 17.00 \\
Densidad (N/ha) & 959 & 1002 & 999 & 989 & 1099 & 993 \\
$\mathrm{G}\left(\mathrm{m}^{2} / \mathrm{ha}\right)$ & 32.66 & 37.38 & 38.32 & 31.36 & 43.28 & 34.21 \\
$\mathrm{~V}\left(\mathrm{~m}^{3} / \mathrm{ha}\right)$ & 237.95 & 281.10 & 309.97 & 231.45 & 325.52 & 273.36 \\
\hline
\end{tabular}

$\mathrm{H}_{\mathrm{m}}=$ Altura media, $\mathrm{DAP} \mathrm{P}_{\mathrm{m}}=$ media de los diámetros medidos a la altura de 1.30, G= Área Basal del rodal,

V= volumen del rodal. 
El clima del área de estudio, según la clasificación de Köppen, es Aw. La caracterización climática se realizó con el climodiagrama de Walter-Lieth (Fig.1a) y el diagrama de balance de hídrico (para una capacidad de campo de 100 $\mathrm{mm} / \mathrm{m}$ ) (Fig.1b) elaborados, utilizando el software PROCLI versión 2.0 (Lago, 2013), con datos de precipitación y temperatura de diez años (2002-2012) proporcionados por la estación meteorológica Isabel Rubio (Pinar del Río). El climodiagrama indicó una precipitación media anual de $1520.1 \mathrm{~mm}$, una temperatura media de 26.6 ${ }^{\circ} \mathrm{C}$ e identificó tres estaciones: una de muy escasas lluvias en el mes de febrero; una de abundantes lluvias que comienza en mayo, se incrementa significativamente a partir de agosto y termina al inicio de noviembre; y una estación seca en los meses de diciembre, enero, marzo y abril. El diagrama de balance hídrico indicó dos estados del suelo: un estado de déficit hídrico que se extiende desde enero hasta principios de agosto y desde finales de noviembre hasta diciembre. Esa temporada puede considerarse favorable para intensas actividades de aprovechamiento forestal ya que la humedad del suelo, uno de los principales factores que reducen la productividad de las máquinas y aumentan la compactación del suelo (Lopes, Sampietro, Lombardi y Dias, 2011; Szymczak et al. 2014), es menor. El segundo estado, caracterizado por un exceso de humedad, coincide con la estación de abundantes lluvias (Fig.1a) y se extiende desde mediados de agosto hasta principios de noviembre.

En la empresa Macurije, la extracción de madera inicia después de la tala semimecanizada, con motosierras, de árboles Pinus caribaea var. caribaea en edad de corte. A esta etapa, le sigue el desrame y troceado de los árboles derribados para obtener trozas cortas ( $3 \mathrm{~m}$ y $4 \mathrm{~m}$ ) y largas $(12 \mathrm{~m})$. Las dimensiones de esas trozas se definen por los tipos de camiones asignados para el transporte posterior de la madera. Luego, las trozas son agrupadas y amarradas con cables a la parte posterior del equipo (skidders o yunta de bueyes) para su extracción del área de tala a los acopiaderos superiores ubicados a una distancia máxima de aproximadamente $300 \mathrm{~m}$ para la tracción mecánica con tractores arrastradores (Trzesniowski, 1993) y $140 \mathrm{~m}$ para la tracción animal (Seixas y Castro, 2014). En los acopiaderos superiores, las trozas son organizadas y clasificadas con base a sus dimensiones con un cargador

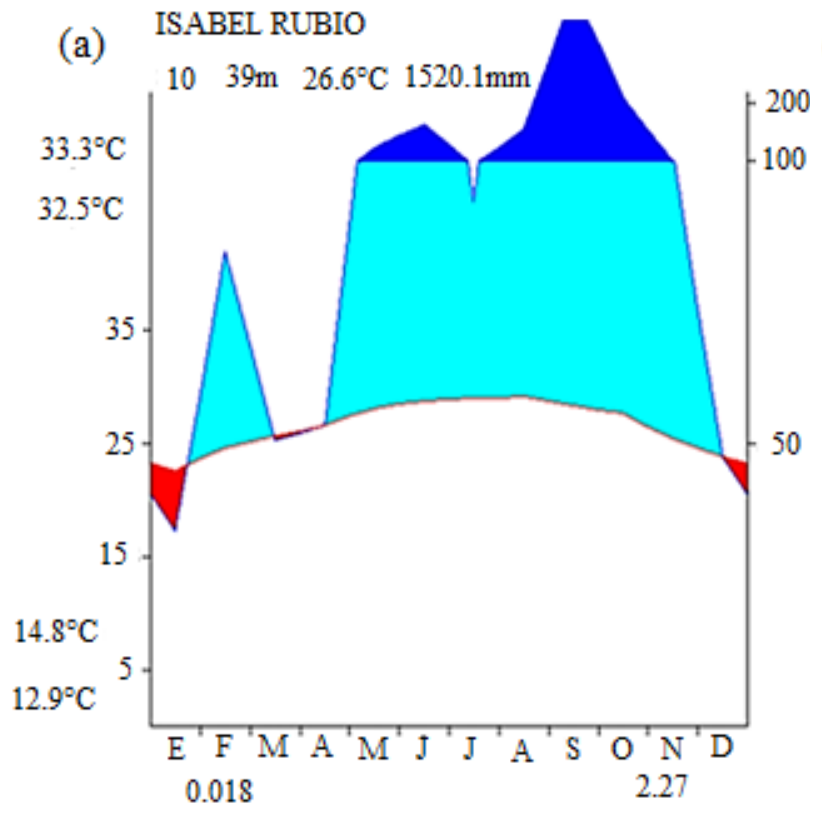

(b)

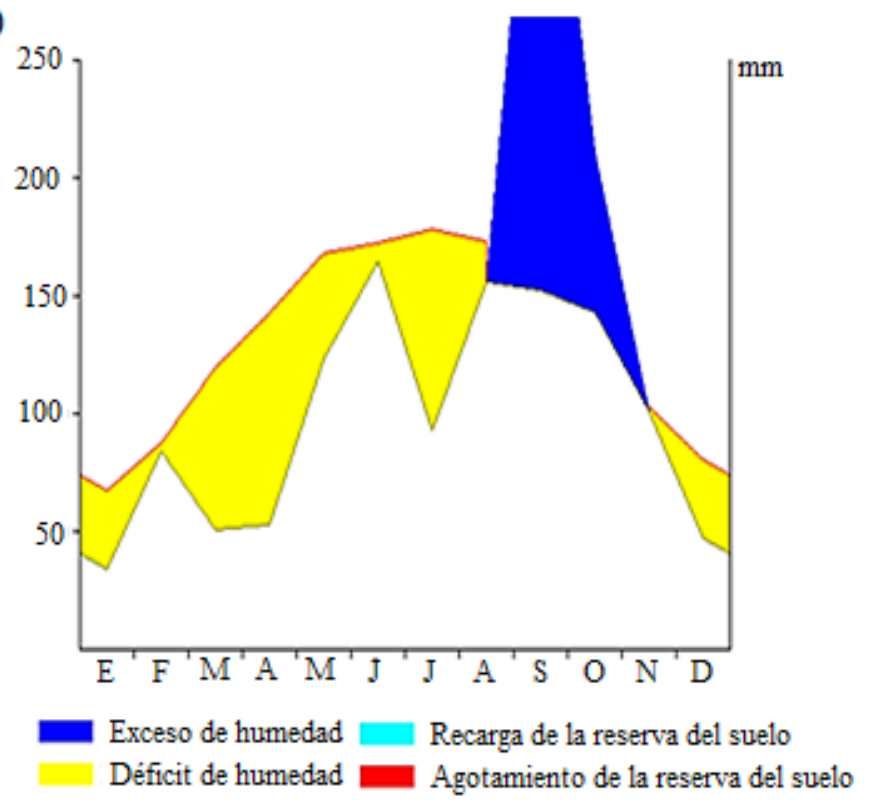

FIgURA 1. Climodiagrama de Walter-Lieth (a) y diagrama de balance hídrico para una capacidad de campo de $100 \mathrm{~mm} / \mathrm{m}$ (b) para el período 2002-2012. 
frontal VOLVO BM L90B cuya principal función es cargar los camiones no autocargables con trozas largas, mientras que los camiones autocargables se autocargan con trozas cortas. Las características de los equipos de extracción, objeto de este estudio, son las siguientes:

John Deere 548E: de origen estadounidense, con un peso de $10.34 \mathrm{t}$, motor de potencia $90.2 \mathrm{~kW}$ (121HP), velocidades permisibles entre $2.4 \mathrm{~km} / \mathrm{h}$ y $26.7 \mathrm{~km} / \mathrm{h}$ y ruedas de neumáticos (Fig. 2a).

J-80A: de origen chino, con un peso de $8.25 \mathrm{t}$, velocidades permisibles entre $2.22 \mathrm{~km} / \mathrm{h}$ y $32.60 \mathrm{~km} / \mathrm{h}$, motor de potencia $73.5 \mathrm{~kW}(110 \mathrm{HP})$ y ruedas de neumáticos (Fig. 2b).

Yunta de bueyes: con un peso promedio de $538 \mathrm{~kg}$ y una edad promedio de siete años, cuenta con un yugo para su control y cables de amarre de la madera como aditamentos. Tienen una experiencia de dos años en la cosecha forestal (Fig. 2c).

Datos experimentales e indicadores de desempeño de las máquinas y equipos de extracción de madera en la empresa forestal integral Macurije

El experimento cubrió los meses de enero a mayo de 2015.

El estudio de tiempo y movimiento se realizó con el método de tiempos individuales en el cual fueron medidos, individualmente, los tiempos de desplazamiento sin carga (Tdsc), desplazamiento con carga (Tdcc), amarre (Ta), desamarre (Td) e interrupción (Ti). También se midieron los volúmenes extraídos y las distancias correspondientes a los tiempos para la determinación de las velocidades medias y de las productividades.

Se evaluaron los sistemas de madera corta ( $3 \mathrm{~m}$ o $4 \mathrm{~m}$ ) y de tronco entero $(12 \mathrm{~m})$. Los indicadores de desempeño o criterios de evaluación utilizados fueron los costos unitarios $\left(\mathrm{USD} / \mathrm{m}^{3}\right.$ ) y las productividades (metro cúbico por hora trabajada, $\mathrm{m}^{3} / \mathrm{h}$ ) calculadas por medio de las fórmulas de las expresiones 01, 02 y 03 (Cándano, 2003; Machado, 2014).

$$
\begin{gathered}
C_{u}=\frac{C E M}{\text { Prod. }} \\
C E M=C_{f}+C_{v}+C_{m o} \\
\text { Prod. }=\frac{V *\left[60-T_{i}\right]}{\frac{d a}{V_{p v}}+T_{a}+\frac{d a}{V_{p c}}+T_{d}}
\end{gathered}
$$

Donde:

$C_{u}=$ costo unitario de extracción o arrastre de madera $\left(\mathrm{USD} / \mathrm{m}^{3}\right)$

$\mathrm{CEM}=$ costo de explotación de una máquina o equipo por hora trabajada (USD/h)

$C_{f}=C_{d}+C_{i}+C_{s}+C_{i m p}=$ costos fijos (costos de propiedad de las máquinas)

$C_{d}=$ costo de depreciación de la máquina $(\mathrm{USD} / \mathrm{h})$

$C_{i}=$ costo de interés de la máquina $(\mathrm{USD} / \mathrm{h})$

$C_{S}=$ costo de seguro de la máquina $(\mathrm{USD} / \mathrm{h})$

$C_{i m p}=$ costo de seguro de la máquina (USD $/ \mathrm{h}$ )

$C_{v}=C_{c}+C_{l}+C_{r m}+C_{o m}=$ costos variables (costos de operación de las máquinas)

$C_{c}=$ costo de combustible $(\mathrm{USD} / \mathrm{h})$

$C_{l}=$ costo de lubricante $(\mathrm{USD} / \mathrm{h})$

$C_{r m}=$ costo de reparación y mantenimiento (USD $/ \mathrm{h}$ )

$C_{o m}=$ costo de otros materiales (USD $/ \mathrm{h}$ )

$C_{m o}=$ costo de mano de obra $(\mathrm{USD} / \mathrm{h})$

Prod. = productividad de la máquina o equipo $\left(\mathrm{m}^{3} / \mathrm{h}\right)$

$V=$ volumen medio de carga de la máquina o equipo por ciclo $\left(\mathrm{m}^{3}\right)$

$T_{i}=$ tiempo de interrupción del trabajo de la máquina o equipo ( $\mathrm{min} / \mathrm{h}$ )

$d_{a}=$ distancia media de arrastre $(\mathrm{m})$

$V_{p v}=$ velocidad de recorrido vacía $(\mathrm{m} / \mathrm{min})$

$T_{a}=$ tiempo de amarre de la madera (min)

$V_{p c}=$ velocidad de recorrido con carga $(\mathrm{m} / \mathrm{min})$

$T_{d}=$ tiempo de desamarre de la madera ( $\mathrm{min}$ )

Las fórmulas complementarias (fórmulas de $C_{d}, C_{i}, C_{S}$, $C_{i m p}, C_{c}, C_{l}, C_{r m}$ y $C_{o m}$ ) se encuentran en Cándano (2003). 

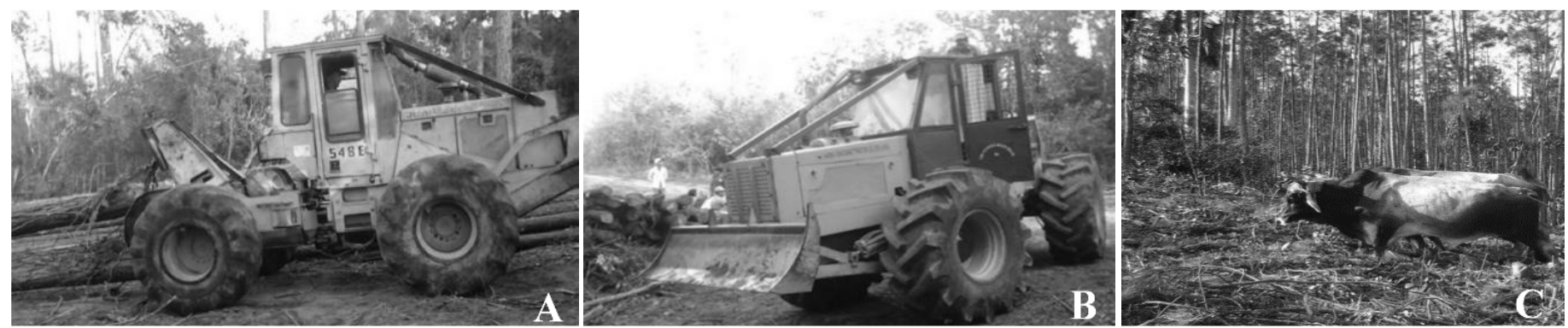

FiguRA 2. Equipos de extracción de madera de la empresa forestal Macurije: A) skidder John Deere 548E; B) skidder J-80A y C) yunta de bueyes.

\section{Análisis de suficiencia muestral}

La muestra inicial (piloto) utilizada en el estudio fue de 45 ciclos operativos para cada uno de los medios de extracción de madera. El número mínimo de ciclos operativos necesarios por equipo de extracción para obtener cierta precisión, prefijada por un error de muestreo máximo permisible de $10 \%$ se determinó con la expresión 04 (Barnes, 1977). Los errores de muestreo se determinaron mediante la expresión 05.

$$
\begin{gathered}
n \geq \frac{t^{2} * C V^{2}}{E^{2}} \\
E_{m}(\%) \leq\left(\frac{S_{\bar{y}} * t}{\bar{Y}}\right) * 100
\end{gathered}
$$

Donde:

$n$ = número mínimo de ciclos operativos necesarios para cada máquina o equipo de extracción

$t=$ valor de $\mathrm{t}$ de student $(\mathrm{gl}=\mathrm{n}-1 ; 1-\alpha=0.95)$

$C V(\%)=$ coeficiente de variación

$E=$ error admisible $(10 \%)$

$E_{m}(\%)=$ error de muestreo de una máquina o equipo de extracción

$S_{\bar{y}}=$ desviación estándar de la media

$\bar{Y}=$ media de la muestra

\section{Experimentos factoriales univariados y multiva- riados en la extracción de madera en la empresa forestal Macurije}

Previamente a la realización de los análisis de varianza (ANOVA y MANOVA), se probaron los supuestos de normalidad y homocedasticidad. Los supuestos de normalidad univariada y multivariada se verificaron con las pruebas de Shapiro-Wilk (Shapiro y Wilk, 1965) y de asimetría y curtosis de Mardia (1970), respectivamente.

En relación con la homocedasticidad, la univariada se analizó con la prueba de Levene (Levene, 1960) y la multivariada con la prueba M de Box (Box, 1949).

Las hipótesis probadas en los arreglos factoriales (univariados o multivariados) fueron:

$\mathrm{H}_{01}$ : No existe diferencia entre costos o productividades generados por el uso de las diferentes máquinas o equipos en la extracción de madera (M);

$\mathrm{H}_{02}$ : No existe diferencia entre costos o productividades registrados en la extracción de madera en las diferentes clases de pendientes $(\mathrm{P})$;

$\mathrm{H}_{03}$ : No existe diferencia significativa entre costos o productividades generados por la adopción de los diferentes sistemas de aprovechamiento $(\mathrm{S})$;

$\mathrm{H}_{04}$ : Las interacciones $\mathrm{M} \times \mathrm{P}, \mathrm{M} \times \mathrm{S}, \mathrm{P} \times \mathrm{S}$ y $\mathrm{M} \times \mathrm{P} \times \mathrm{S}$ no son significativas.

\section{Experimento factorial univariado}

El experimento univariado siguió un diseño al azar en bloques con arreglo factorial en el esquema $\mathrm{M} \times \mathrm{P} \times \mathrm{S}(3 \times 3$ $\times 2$ ). Los bloques fueron: rodales de 21 años (bloque $\mathrm{I}$ ) (rotación adoptada en la empresa Macurije) y rodales de 32 años (bloque II) (rotación propuesta por Medel, Equino, Lazo y Guera, 2011 y Guera, Silva, Ferreira, Álvarez y Barrero, 2019) con el propósito de controlar posibles efectos de la variación de la edad de rotación en las 
productividades y costos de extracción de madera. La eficiencia de bloque se analizó para cada una de las variables (costo unitario y productividad) con la expresión 06 (Kutner, Nachtsheim, Neter y Li, 2005).

$$
E_{B}=\frac{\left(n_{b}-1\right) C M B+n_{b}(r-1) C M R}{\left(n_{b} r-1\right) C M R}
$$

Donde:

$E_{B}=$ eficiencia de bloques

$C M B=$ cuadrado medio de bloques

$C M R=$ cuadrado medio de residuos

$r=\quad$ número de tratamientos

$n_{b}=$ número de bloques

$E_{B}=1$ indica que cualquiera de los dos diseños (diseño completamente al azar y diseño al azar en Bloques) puede usarse

$E_{B}<1$ indica la ineficiencia de los bloques, recomendando el uso del diseño completamente al azar

$E_{B}>1$ indica la eficiencia de los bloques, recomendando su uso

Los niveles de los tres factores supracitados fueron: el factor M (Maquinaria) con tres niveles: 1) skidder J-80A, 2) skidder John Deere 548E, 3) yunta de bueyes; el factor $\mathrm{P}$ (Pendiente) con tres niveles: 1) $\mathrm{P}<8 \%, 2) 8 \% \leq \mathrm{P} \leq 16 \%$, 3) $16 \%<\mathrm{P}<25 \%$ y el Factor $\mathrm{S}$ (método o sistema de aprovechamiento) con dos niveles: 1) troncos enteros de 12 $\mathrm{m}, 2$ ) madera corta de 3 o $4 \mathrm{~m}$.

El modelo matemático para el arreglo factorial univariado $3 \times 3 \times 2$ del diseño experimental en bloques (DCB) fue la expresión 07:

$$
\begin{aligned}
& Y_{i j k l}=\mu+\alpha_{i}+\beta_{j}+\gamma_{k}+(\alpha \beta)_{i j}+(\alpha \gamma)_{i k}+ \\
& (\beta \gamma)_{j k}+\delta_{i j k}+b_{l k}+e_{i j k l}
\end{aligned}
$$

Donde:

$\mathrm{Y}_{\mathrm{ijkl}}=$ valores observados de la variable respuesta (productividad o costo) en el $i$-ésimo nivel del factor $\mathrm{M}, j$-ésimo nivel del factor $\mathrm{P}, k$-ésimo nivel del factor $\mathrm{S}$ del $l$-ésimo bloque

$\mu$ : una constante (media general)

$\alpha_{\mathrm{i}}$ : efecto del $i$-ésimo nivel del factor $\mathrm{M}$ $\beta_{j}: \quad$ efecto del $j$-ésimo nivel del factor $\mathrm{P}$

$\gamma_{\mathrm{k}}$ : efecto del k-ésimo nivel del factor $\mathrm{S}$ $(\alpha \beta)_{\mathrm{ij}}$ : efecto de la interacción entre M y $\mathrm{P}$ $(\alpha \gamma)_{\mathrm{ik}}$ : efecto de la interacción entre $\mathrm{M}$ y $\mathrm{S}$ $(\beta \gamma)_{\mathrm{jk}}$ : efecto de la interacción entre P y $\mathrm{S}$ $\delta_{\mathrm{ijk}}$ : efecto de la interacción entre M, P y $\mathrm{S}$ $\mathrm{b}_{\mathrm{lk}}$ : efecto del bloque 1

$\mathrm{e}_{\mathrm{ijkl}}$ : error experimental

En caso de existir diferencia significativa, las comparaciones por pares se realizaron por medio de la prueba de Tukey $(\alpha=0.05)$ (Tukey, 1949). Los análisis se realizaron con el software SPSS versión 20.

\section{Experimento factorial multivariado}

Para el estudio comparativo de los diferentes medios de extracción en los diferentes escenarios, basándose simultáneamente en las productividades y en los costos unitarios, se realizó un experimento factorial multivariado adoptando la misma estructura anteriormente descrita para el experimento univariado. El modelo matemático del arreglo factorial multivariado $3 \times 3 \times 2$ fue (Expresión 08):

$$
\begin{aligned}
& Y_{i j k l r}=\mu_{r}+\alpha_{i r}+\beta_{j r}+\gamma_{k r}+(\alpha \beta)_{i j r}+ \\
& (\alpha \gamma)_{i k r}+(\beta \gamma)_{j k r}+\delta_{i j k r}+b_{l k r}+e_{i j k l r}
\end{aligned}
$$

Donde:

$\mathrm{Y}_{\mathrm{ijklr}}$ : valor observado de la r-ésima variable respuesta en el i-ésimo nivel del factor $\mathrm{M}$, j-ésimo nivel del factor $\mathrm{P}$, k-ésimo nivel del factor $\mathrm{S}$, en el l-ésimo bloque

$\mu_{\mathrm{r}}$ : una constante (media general) de la r-ésima variable

$\alpha_{i r}$ : efecto del i-ésimo nivel del factor $M$ en la r-ésima variable

$\beta_{\mathrm{j} \text { : }}$ efecto del j-ésimo nivel del factor $\mathrm{P}$ en la r-ésima variable

$\gamma_{\mathrm{kr}}$ : efecto del k-ésimo nivel del factor $\mathrm{S}$ en la r-ésima variable

$(\alpha \beta)_{\mathrm{ijr}}$ : efecto de la interacción entre M y P en la r-ésima variable 
$(\alpha \gamma)_{\mathrm{ikr}}$ : efecto de la interacción entre M y S en la r-ésima variable

$(\beta \gamma)_{\mathrm{jkr}}$ : efecto de la interacción entre P y S en la r-ésima variable

$\delta_{\mathrm{ijkr}}$ : efecto de la interacción entre M, P y S en la r-ésima variable

$\mathrm{b}_{\mathrm{lkr}}$ : efecto del bloque 1 en la r-ésima variable

$\mathrm{e}_{\mathrm{ijklr}}$ : error aleatorio asociado

El análisis de varianza del experimento factorial multivariado se realizó con el software SAS (SAS, 1999) que dispone de las pruebas de lambda de Wilks, traza de Pillai, traza de Lawley-Hotelling y la Mayor Raíz de Roy para las comparaciones. En los casos de rechazo de las hipótesis nulas ( $\mathrm{p}$-valor $<0.05$ ), las comparaciones múltiples entre vectores de medias se realizaron con la prueba $\mathrm{T}^{2} \mathrm{de}$ Hotelling (Hotelling, 1931) (Expresiones 09 y 10), análogo multivariado de la prueba t de Student, testando la hipótesis nula, $H_{0}: \mu_{1}=\mu_{2}$, siendo $\mu_{1}$ y $\mu_{2}$ los vectores de medias.

$$
\begin{gathered}
T^{2}=(\bar{X}-\bar{Y})^{T}\left[S\left(\frac{1}{n_{x}}+\frac{1}{n_{y}}\right)\right]^{-1}(\bar{X}-\bar{Y}) \\
S=\frac{\left(n_{x}-1\right) S_{X}+\left(n_{y}-1\right) S_{Y}}{\left(n_{x}-1\right)+\left(n_{y}-1\right)}
\end{gathered}
$$

Donde:

$S_{X}$ y $S_{Y}=$ matrices de covarianza de $X$ y $Y$, respectivamente $\bar{X}_{\text {y }} \bar{Y}=$ medias de $X_{\text {y }} \mathrm{Y}$, respectivamente

$\mathrm{n}_{\mathrm{x}}$ y $\mathrm{n}_{\mathrm{y}}$ son los tamaños de muestra de $\mathrm{X}$ y $\mathrm{Y}$, respectivamente

Las significaciones se determinaron aproximando la prueba $\mathrm{T}^{2}$ de Hotelling a la prueba $F$ de la siguiente manera (Expresión 11):

$$
F=\frac{n-k}{k(n-1)} T^{2} \sim F(k, n-k)
$$

Donde:

$\mathrm{n}=\mathrm{n}_{\mathrm{x}}+\mathrm{n}_{\mathrm{y}}-1=$ grados de libertad

$\mathrm{k}=$ número de variables

\section{RESUltadOS}

\section{Suficiencia muestral y estudios de tiempos y movimientos de las máquinas y equipos en la extracción de madera en la empresa forestal Macurije}

A partir de las muestras iniciales (pilotos) de 45 ciclos operativos para cada uno los medios de extracción, se detectaron errores muestrales de $3.01 \%, 3.97 \%$ y $3.54 \%$ correspondientes a los skidders J-80A, John Deere 548E y la yunta de bueyes, respectivamente. Estos errores, siendo inferiores al error permisible de 10\%, las muestras iniciales ( $\mathrm{n}=45)$ se consideraron definitivas y suficientes para atender la precisión requerida en el estudio, pues los tamaños mínimos para un error de 10\%, fueron 29, 17 y 23 para los skidders J-80A y John Deere y la yunta de bueyes, respectivamente.

Los resultados de los estudios de tiempos y movimientos (Tabla 2) indicaron porcentajes similares en la distribución del tiempo en cada elemento del ciclo de arrastre de trozas en los skidders. En las yuntas de bueyes, los tiempos de interrupciones también fueron los mayores, seguidos de los tiempos de viajes, siendo esto característico de la extracción animal.

\section{Productividades y costos de explotación de madera en la empresa forestal Macurije}

Los costos de explotación (Tabla 3; Fig. 3) indicaron, como podría esperarse, que la menos onerosa fue la yunta de bueyes con solo $1.34 \mathrm{USD} / \mathrm{h}$, pero también fue la que presentó la menor productividad por hora trabajada (3.95 $\mathrm{m}^{3} / \mathrm{h}$ ). El tractor chino fue el más costoso con un costo operacional de $29.55 \mathrm{USD} / \mathrm{h}$ y una productividad intermedia de $15.98 \mathrm{~m}^{3} / \mathrm{h}$. A diferencia de la yunta de bueyes en la que el componente principal de los costos fue el de mano de obra, en ambos skidders, los componentes principales fueron los costos operacionales (costos variables) (Fig. 3). 
TABLA 2. Distribución de tiempos entre los diferentes componentes del ciclo de extracción de madera para una distancia de 150 m.

\begin{tabular}{cccc}
\hline \multirow{2}{*}{ Componentes del ciclo } & \multicolumn{3}{c}{ Tiempos medios (min) } \\
\cline { 2 - 4 } & John Deere 548E & J-80A & Yunta de bueyes \\
\hline Tiempo de viaje vacío & $1.56(11.76 \%)$ & $1.84(11.75 \%)$ & $5.56(22.62)$ \\
Tiempo de viaje cargado & $1.76(13.27 \%)$ & $2.32(14.81 \%)$ & $7.00(28.48)$ \\
Tiempo de amarre de las trozas & $3.50(26.40 \%)$ & $4.44(28.35 \%)$ & $2.26(9.19)$ \\
Tiempo de desamarre de las trozas & $2.62(19.76 \%)$ & $2.48(15.84 \%)$ & $1.22(4.96)$ \\
Tiempo de interrupciones & $3.82(28.81 \%)$ & $4.58(29.25 \%)$ & $8.54(34.74)$ \\
\hline Tiempo total & $13.26(100 \%)$ & $15.66(100 \%)$ & $24.58(100 \%)$ \\
\hline
\end{tabular}

TABLA 3. Productividades y costos de los medios de extracción de madera de P. caribaea var. caribaea para una distancia de arrastre de 150 m.

\begin{tabular}{cccc}
\hline Medios de extracción de madera & $\begin{array}{c}\text { PROD } \\
\left(\mathrm{m}^{3} / \mathrm{h}\right)\end{array}$ & $\begin{array}{c}\text { CEM } \\
(\mathrm{USD} / \mathrm{h})\end{array}$ & $\begin{array}{c}\mathrm{Cu}_{u} \\
(\text { USD/m }\end{array}$ \\
\hline John Deere 548E & 17.20 & 17.48 & 1.02 \\
J-80A & 15.98 & 29.55 & 1.85 \\
Yunta de bueyes & 3.95 & 1.343 & 0.34 \\
\hline
\end{tabular}

PROD =productividad por hora trabajada $\left(\mathrm{m}^{3} / \mathrm{h}\right) ; \mathrm{CEM}=$ costo de explotación de una máquina o equipo por hora trabajada (USD/h); C $u=$ costo unitario de extracción

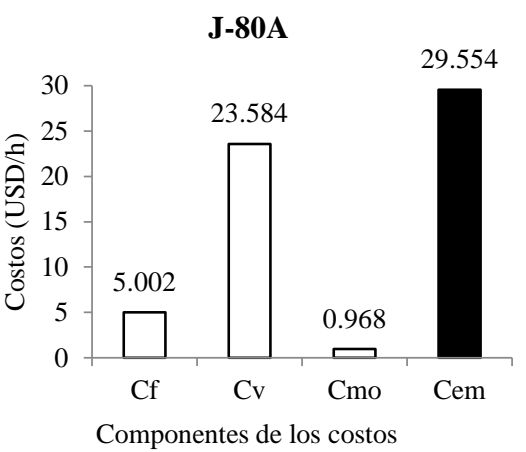

Componentes de los costos
John Deere 548 E

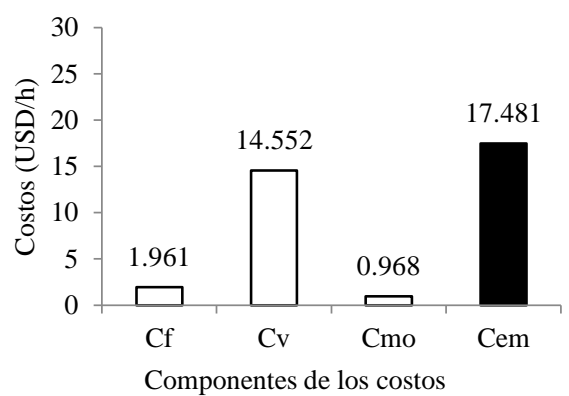

Yunta de bueyes

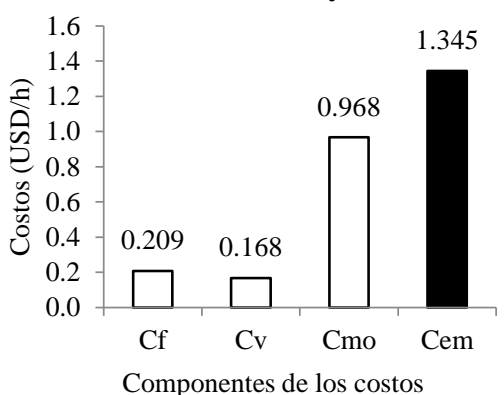

Figura 3. Componentes de los costos (fijos-Cf, variables- $\mathrm{Cv}$, mano de obra-Cmo; $\mathrm{Cem}=\mathrm{Cf}+\mathrm{Cv}+\mathrm{Cmo}$ ) de extracción de madera de P. caribaea var. caribaea, Pinar del Rio, Cuba.

Los resultados de la prueba de Shapiro-Wilk indicaron que el supuesto de normalidad univariada se cumplió en ambas variables: costo unitario $(\mathrm{SW}=0.936 ; \mathrm{p}$-valor $=0.089>$ $0.05)$ y productividad $(\mathrm{SW}=0.900 ; \mathrm{p}$-valor $=0.053>0.05)$. El resultado de la prueba de asimetría y curtosis de Mardia indicó también la satisfacción de la normalidad multivariada $\left(\chi^{2}=0.188 ; \mathrm{p}\right.$-valor $\left.=0.991>0.05\right)$. En cuanto a la homocedasticidad, los resultados de la prueba de Levene indicaron el cumplimiento de este supuesto tanto para el costo $(\mathrm{F}=6.667$; p-valor $=0.081>0.05)$ como para la productividad $(\mathrm{F}=6.702 ; \mathrm{p}$-valor $=0.061>0.05)$. No obstante, la significancia de la prueba $\mathrm{M}$ de Box $(\mathrm{M}=$ 33.533; $\mathrm{F}=5.098$; $\mathrm{p}$-valor $<0.05$ ) indicó presencia de heterocedasticidad multivariada en las matrices de varianzacovariancia. Este supuesto es raramente satisfecho en la práctica (O'rourke, Hatcher y Stepanski, 2005) y su 
violación tiene un impacto mínimo si los grupos comparados tienen el mismo tamaño (Hair Jr, Anderson, Tatham y Black, 2005; Maroco, 2007) como es el caso en el presente estudio. Bray y Maxwell (1985) recomiendan en esta situación (tamaños de muestras iguales), el uso de la traza de Pillai-Bartlett debido a su mayor robustez frente a esa violación.

\section{Experimento factorial univariado para las variables dependientes costo y productividad}

Los resultados indicaron que, para la variable dependiente costo, los tres factores $\mathrm{M}, \mathrm{P}, \mathrm{S}$ y la interacción $\mathrm{M} \times \mathrm{P}$ fueron significativos. Para la variable dependiente productividad, los resultados fueron similares, con excepción del factor $\mathrm{S}$ que no fue significativo, lo que indica que no existe diferencia entre las productividades de extracción con los sistemas de madera corta o de troncos enteros (Tabla 4).

Los resultados de la tabla 5 indican que no hay diferencia significativa entre las productividades de los medios de extracción en los dos sistemas de aprovechamiento, aunque el sistema de troncos entero haya presentado valores numéricamente mayores. Con relación al costo, tanto los skidders como las yuntas de bueyes presentaron menor costo en el sistema de tronco entero.

Para ambas variables dependientes, los resultados de la prueba de Tukey aplicada a las interacciones significativas se encuentran en la tabla 6.

TABLA 4. Análisis de varianza (ANOVAs) para los arreglos factoriales univariados $3 \times 3 \times 2$

\begin{tabular}{|c|c|c|c|c|c|c|c|c|}
\hline \multirow{2}{*}{$\begin{array}{c}\text { Variables } \\
\text { dependientes } \\
\begin{array}{c}\text { Fuentes de } \\
\text { variación }\end{array}\end{array}$} & \multicolumn{4}{|c|}{ Productividad } & \multicolumn{4}{|c|}{ Costo unitario } \\
\hline & $G L$ & $C M$ & $F$ & $\operatorname{Pr}>F$ & $G L$ & $C M$ & $F$ & $\operatorname{Pr}>F$ \\
\hline Bloque & 1 & 22.753 & 27.90 & $<0.0001$ & 1 & 0.198 & 25.29 & $<0.0001$ \\
\hline Tratamiento & 17 & 24.683 & 30.27 & $<0.0001$ & 17 & 1.325 & 169.16 & $<0.0001$ \\
\hline Maquinaria (M) & 2 & 353.475 & 433.49 & $<0.0001$ & 2 & 11.400 & 1455.79 & $<0.0001$ \\
\hline Pendiente (P) & 2 & 43.781 & 53.69 & $<0.0001$ & 2 & 1.765 & 225.42 & $<0.0001$ \\
\hline $\begin{array}{c}\text { Sistema de } \\
\text { aprovechamiento (S) }\end{array}$ & 1 & 7.765 & 9.52 & 0.0670 & 1 & 0.178 & 22.71 & 0.0002 \\
\hline$M \times P$ & 4 & 6.007 & 7.37 & $<0.0001$ & 4 & 0.162 & 20.66 & $<0.0001$ \\
\hline$M \times S$ & 2 & 0.676 & 0.83 & 0.4533 & 2 & 0.021 & 2.67 & 0.0981 \\
\hline$P \times S$ & 2 & 1.614 & 1.98 & 0.1688 & 2 & 0.002 & 0.33 & 0.7262 \\
\hline$M \times P \times S$ & 4 & 0.051 & 0.06 & 0.9922 & 4 & 0.002 & 0.28 & 0.8850 \\
\hline Error & 17 & 0.815 & & & 17 & 0.008 & & \\
\hline Total & 35 & & & & 35 & & & \\
\hline
\end{tabular}

CM=cuadrado medio; GL= grados de libertad.

TABla 5. Productividades y costos de los medios de extracción de madera de $P$. caribaea var. caribaea en diferentes sistemas de aprovechamiento

\begin{tabular}{|c|c|c|c|c|}
\hline \multirow{2}{*}{ Medio de extracción } & \multicolumn{2}{|c|}{$\begin{array}{l}\text { Productividad por hora trabajada } \\
\qquad\left(\mathrm{m}^{3} / \mathrm{h}\right)\end{array}$} & \multicolumn{2}{|c|}{$\begin{array}{l}\text { Costo unitario } \\
\left(U S D / m^{3}\right)\end{array}$} \\
\hline & \multicolumn{4}{|c|}{ Sistemas de aprovechamiento } \\
\hline John Deere 548E & 17.97 a & $16.65 \mathrm{a}$ & $1.01 \mathrm{~b}$ & $1.17 \mathrm{a}$ \\
\hline Yunta de bueyes & 3.98 a & $3.41 \mathrm{a}$ & $0.32 \mathrm{~b}$ & 0.59 a \\
\hline
\end{tabular}

Letras distintas indican diferencias significativas $(p<0.05)$ para costo o productividad por la prueba de Tukey ( $\alpha=0.05)$ entre los sistemas de aprovechamiento. 
TABLA 6. Prueba de Tukey para la interacción $\mathrm{M} \times \mathrm{P}$ para las variables productividad y costo

\begin{tabular}{lccc}
\hline \multicolumn{4}{c}{ Variable dependiente: productividad } \\
\hline \multirow{2}{*}{ Maquinaria } & \multicolumn{3}{c}{ Pendiente del terreno } \\
\cline { 2 - 3 } J-80A & $P<8 \%$ & $8 \% \leq P \leq 16 \%$ & $16 \%<P<25 \%$ \\
John Deere 548 E & $13.1025 \mathrm{bA}$ & $10.1650 \mathrm{bB}$ & $8.0425 \mathrm{bC}$ \\
Yunta de bueyes & $15.0825 \mathrm{aA}$ & $12.1375 \mathrm{aB}$ & $9.7150 \mathrm{aC}$ \\
\hline & $2.7000 \mathrm{cA}$ & $1.9250 \mathrm{cA}$ & $1.7200 \mathrm{cA}$ \\
\hline \multirow{2}{*}{ Maquinaria } & \multicolumn{4}{c}{ Variable dependiente: costo } \\
\hline J-80A & $\mathrm{P}<8 \%$ & Pendiente del terreno \\
John Deere 548E & $2.0075 \mathrm{aC}$ & $8 \% \leq \mathrm{P} \leq 16 \%$ & $3.2025 \mathrm{aA}$ \\
Yunta de bueyes & $1.1275 \mathrm{bC}$ & $2.5575 \mathrm{aB}$ & $1.7925 \mathrm{bA}$ \\
\hline
\end{tabular}

Las medias seguidas de la misma letra (minúscula en las columnas y mayúscula en las líneas) no difieren entre sí para la prueba de Tukey ( $\alpha=0.05)$.

\section{Experimento factorial multivariado para el con- junto "costo-productividad"}

La correlación moderada, sin embargo, significativa, entre las variables costo unitario y productividad $(\mathrm{r}=0.404 ; p$ valor $=0.014<0.05)$ indica que es factible utilizar el análisis de varianza multivariado (MANOVA), utilizado con el intuito de resolver la divergencia entre los resultados encontrados en los enfoques univariados. De acuerdo con los resultados de este, todos los factores y la interacción $\mathrm{M} \times \mathrm{P}$ fueron significativos (Tabla 7).

Los resultados de la prueba $\mathrm{T}^{2}$ de Hotelling para vectores de medias en la interacción MxP indicaron una alta influencia de esta en el conjunto costo-productividad (Tabla 8).

\section{DISCUSIÓN}

Los resultados de los estudios de tiempos y movimientos (Tabla 2) indicaron que las interrupciones llevaron el mayor porcentaje de tiempo en los tres medios de extracción y los tiempos de amarre y desamarre fueron superiores a los tiempos de los viajes. Esta distribución del tiempo está en correspondencia con los resultados de Lopes y Diniz (2015) y es típica de skidders de cable como los evaluados en el presente estudio. Los altos porcentajes de esos tiempos de amarre y desamarre se atribuyen principalmente a la dificultad que encuentran los ayudantes en el amarre y desamarre de la madera con los cables antes de iniciar el viaje cargado. Estos resultados indican que los trabajos a ser desarrollados para la mejora de la productividad de las máquinas tienen que ser orientados a minimizar los tiempos de interrupciones en todos los medios de extracción y los tiempos de amarre y desamarre en los skidders.

Los resultados de los costos y productividades indican que el skidder John Deere es la mejor alternativa para la extracción semimecanizada de la madera por tener la mayor productividad $\left(17.20 \mathrm{~m}^{3} / \mathrm{h}\right)$ y el menor costo unitario $(1.02$ $\mathrm{USD} / \mathrm{m}^{3}$ ) de los dos skidders (Tabla 3). La yunta de bueyes presentó costos muy menores a los de los skidders y por ende es la opción más rentable. Sin embargo, su baja productividad hace impracticable su uso exclusivo ya que, para asegurar el abasto continuo de madera en las unidades de transformación primaria, eso implicaría aumentar significativamente su cantidad para compensar esa baja productividad. Sería una alternativa viable para la extracción de madera en pendientes altas y/o en regímenes de producción lenta (cuando no hay limitación de tiempo para cumplir con los planes de producción). Otra opción factible es asociarla a otros medios de extracción (tractores) para aumentar la productividad sin incrementar significati- 
vamente los costos operacionales. En este sentido los resultados de Cándano (1998) indicaron que, al combinar la yunta de bueyes utilizándolas para formar pilas de madera antes de su extracción con tractores, se registró una reducción media de 10.88 min en el tiempo total del ciclo de los tractores, lo que generó un incremento de la productividad.

Los resultados del análisis de eficiencia de bloques $\left(E_{B(\operatorname{Prod})}=13.71\right.$ y $\left.E_{B(\text { costo })}=1.68\right)$ revelaron mayor eficiencia del diseño aleatorio en bloques para ambas variables (costo y productividad). La productividad en el bloque II (media $=9.08 \mathrm{~m}^{3} / \mathrm{h}$ ) fue superior a la del bloque I $\left(\right.$ media $=7.49 \mathrm{~m}^{3} / \mathrm{h}$ ). Estos resultados están en concordancia con los obtenidos por Santos y Machado (1995), Holtzscher y Lanford (1997) y Elliasson (1999) según los cuales rodales con árboles de pequeños diámetros requieren un mayor tiempo de operación por metro cúbico que rodales con mayores diámetros medios, proporcionando así menores productividades. La alta correlación positiva entre la edad y el diámetro de los árboles explica la similitud de la influencia de ambas variables sobre la productividad. Los costos unitarios también indicaron mayor viabilidad del bloque II (32 años) que presentó una media de $1.49 \mathrm{USD} / \mathrm{m}^{3}$ contra 1.64 $\mathrm{USD} / \mathrm{m}^{3}$ para el bloque I (21 años). Este resultado está en consonancia con los de Santos y Machado (1995) y Holtzscher y Lanford (1997) según los cuales los costos de producción, incluidos los de extracción, son inversamente proporcionales a las dimensiones de los árboles que están, a su vez, altamente correlacionadas con la edad estos.

TABLA 7. Efecto de los factores e interacciones sobre el conjunto "costo-productividad"

\begin{tabular}{lccc}
\hline \multirow{2}{*}{ Factores e interacciones } & \multicolumn{3}{c}{ Traza de pillai $(t p)$} \\
\cline { 2 - 4 } & Valor & $F$ & Pr> F \\
\hline Maquinaria (M) & 1.94 & 279.78 & $<0.0001$ \\
Pendiente (P) & 1.19 & 12.61 & $<0.0001$ \\
Sistema de aprovechamiento (S) & 0.57 & 10.83 & 0.0011 \\
M×P & 1.40 & 9.95 & $<0.0001$ \\
M×S & 0.36 & 1.88 & 0.1372 \\
P×S & 0.35 & 1.80 & 0.1516 \\
M×P×S & 0.14 & 0.31 & 0.9561 \\
\hline
\end{tabular}

TABLA 8. Prueba $\mathrm{T}^{2}$ de Hotelling entre los pares de vectores de medias para la interacción $\mathrm{M} \times \mathrm{P}$

\begin{tabular}{|c|c|c|c|c|c|c|c|c|c|}
\hline & $M_{1} P_{l}$ & $M_{1} P_{2}$ & $M_{1} P_{3}$ & $M_{2} P_{1}$ & $M_{2} P_{2}$ & $M_{2} P_{3}$ & $M_{3} P_{1}$ & $M_{3} P_{2}$ & $M_{3} P_{3}$ \\
\hline$M_{1} P_{l}$ & -- & * & * & * & \# & \# & * & \# & \# \\
\hline$M_{1} P_{2}$ & & -- & * & \# & * & \# & \# & * & \# \\
\hline$M_{1} P_{3}$ & & & -- & $\#$ & $\#$ & * & \# & \# & * \\
\hline$M_{2} P_{1}$ & & & & -- & NS & * & * & \# & \# \\
\hline$M_{2} P_{2}$ & & & & & -- & * & \# & * & \# \\
\hline$M_{2} P_{3}$ & & & & & & -- & \# & \# & * \\
\hline$M_{3} P_{1}$ & & & & & & & -- & * & * \\
\hline$M_{3} P_{2}$ & & & & & & & & -- & NS \\
\hline$M_{3} P_{3}$ & & & & & & & & & -- \\
\hline
\end{tabular}

* Significativo ( $<$ < 0.05); NS = No Significativo ( $>$ > 0.05); \# = comparación sin interés. M1, M2 y M3 son los medios de extracción J-80A, John Deere 548E y Yunta de bueyes, respectivamente. $P_{1}, P_{2}$ y $P_{3}$ son las tres pendientes $P_{1}<8 \%, 8 \% \leq P_{2} \leq 16 \%$ y $16 \%<P_{3}<25 \%$, respectivamente. 
En los análisis univariados, el factor $\mathrm{S}$ no fue significativo para la variable dependiente productividad (Tabla 4). La no significancia de este factor es atribuible a dos aspectos importantes: 1) una mayor facilidad de organización de las trozas cortas en los carriles de arrime, lo que implica un ahorro de tiempo y 2) la extracción de mayor número de trozas cortas por ciclo. En una distancia de arrastre de $150 \mathrm{~m}$, en el sistema de troncos enteros, los skidders arrastraron en promedio cinco troncos/ciclo (por cuestiones operativas y de movilidad), sea un volumen medio de $3.80 \mathrm{~m}^{3}$ en ciclos de aproximadamente $12.69 \mathrm{~min}$ para el skidder John Deere 548E y 14.19 min para J-80A. Las yuntas de bueyes extrajeron aproximadamente $1.62 \mathrm{~m}^{3}$ (entre 2 y 3 troncos) en ciclos de duración media de 24.42 min Ya en el sistema de madera corta $(3 \mathrm{~m}$ o $4 \mathrm{~m}$ ), se extraen por ciclo hasta siete trozas y en ocasiones, en terrenos menos inclinados, hasta ocho, sea un promedio de $3.30 \mathrm{~m}^{3}$ en ciclos con duraciones medias de 11.90 min para John Deere 548E y 13.28 min para J-80A. La yunta de bueyes, en este sistema, operó en ciclos de duración media de $23.75 \mathrm{~min}$, extrayendo aproximadamente 3 trozas correspondientes a $1.35 \mathrm{~m}^{3}$ por ciclo.

Para la variable costo, los resultados indicaron que existe diferencia significativa entre los costos de los dos sistemas de aprovechamiento evaluados (Tabla 4), siendo los costos unitarios de los tres medios de extracción en el sistema de madera corta significativamente mayores a los registrados en el sistema de tronco entero (Tabla 5). Se percibe una divergencia entre los resultados del factor $\mathrm{S}$, ya que los resultados indican que este no afecta la productividad, pero sí al costo de extracción (Tabla 4). Cualquier decisión tomada con respecto a qué sistema de aprovechamiento adoptar, no será libre de subjetividad, ya que dependerá de la variable que se considere en la toma de decisión.

Para la variable dependiente productividad, la prueba de Tukey aplicada a la única interacción significativa $(\mathrm{M} \times \mathrm{P})$ permitió notar que, en todas y cada una de las clases de pendiente, existió diferencia significativa entre las productividades de las máquinas evaluadas, siendo la de mayor productividad el John Deere (Tabla 6). En cuanto al desempeño de cada una de las máquinas a lo largo de las pendientes, los skidders tuvieron un desempeño diferenciado, presentando una productividad mayor en la clase de menor pendiente. Estos resultados corresponden con los de

Behjou, Majnounian, Namiranian y Dvořák (2008) y Leite et al. (2014), según los cuales la pendiente del terreno es uno de los factores más influyentes en la productividad de las máquinas forestales. Igual que en el presente estudio, estos autores encontraron que el aumento de la pendiente del terreno provoca una disminución significativa en la productividad de las máquinas. En cambio, aunque la yunta de bueyes presentó una tendencia similar, no hubo diferencia significativa entre sus desempeños a lo largo de las tres clases de pendiente (Tabla 6). Eso explica, en parte, la razón por la cual la tracción animal, en este caso por la yunta de bueyes, es utilizado en altas pendientes donde las extracciones de madera mecanizadas y semimecanizadas son impracticables.

En cuanto a la variable costo, el resultado de la prueba de Tukey (Tabla 6) indicó que, en todas las clases de pendiente, hubo diferencia significativa entre los costos unitarios de los medios de extracción de madera, siendo el skidder John Deere 548E, el menos costoso. En relación con los desempeños a lo largo de las tres pendientes, se percibió que los tres medios de extracción presentaron costos unitarios estadísticamente diferentes (Tabla 6), con una tendencia de aumento de los costos unitarios con el incremento de la pendiente. Esos costos se ven afectados por la mayor pendiente del terreno en dos formas: una primera relacionada con su crecimiento debido a la disminución de la productividad y una segunda relacionada con el aumento de los costos de explotación de los skidders debido al aumento sustancial del consumo de combustible en pendientes altas, como resultados de la necesidad de mayores potencias en la realización de las operaciones.

De los resultados del enfoque multivariado (Tabla 7), se deduce que, tanto la maquinaria utilizada en la extracción de la madera como el método de aprovechamiento utilizado y las pendientes en las que se extrae la madera, influyen 
significativamente en el vector de medias costoproductividad.

Las diferencias multivariadas (significancia desde los puntos de vista económico y productivo) encontradas entre los sistemas de aprovechamiento (factor $\mathrm{S}$ ) se atribuyen a los menores costos del sistema de troncos enteros (Tabla 5) considerado más viable. Estos resultados coinciden con los encontrados por Machado y Lopes (2000) en plantaciones de Eucalipto y los de Mousavi (2012) en rodales mixtos, cuyos resultados también indicaron mayor viabilidad del sistema de troncos enteros.

Similarmente a los resultados de los análisis univariados, se observó que apenas la interacción $\mathrm{M} \times \mathrm{P}$ fue significativa y todas las interacciones que involucraron al factor $\mathrm{S}$ no fueron significativas (Tabla 7). Esto indica que una óptima combinación de los factores $\mathrm{M}$ y $\mathrm{P}$ puede inhibir el efecto del factor $\mathrm{S}$ en el conjunto costoproductividad y los sistemas de aprovechamiento de tronco entero y madera corta podrán ser adoptados sin perjudicar la rentabilidad de la producción.

Las significancias encontradas en las comparaciones por pares de vectores de medias (Tabla 8) indicaron un efecto significativo simultáneo de la interacción $\mathrm{M} \times \mathrm{P}$ en las productividades y los costos unitarios de extracción de madera. Se observó una diferencia significativa entre los desempeños (costo-productividad) del skidder J-80A en las tres clases de pendiente. John Deere presentó desempeños similares en las dos primeras clases de pendientes $\left(\mathrm{P}_{1}\right.$ y $\left.\mathrm{P}_{2}\right)$, siendo estos dos superiores al de la tercera clase $\left(\mathrm{P}_{3}\right)$. En cuanto a las yuntas de bueyes, su desempeño en la primera clase de pendientes $\left(\mathrm{P}_{1}\right)$ fue superior a los de las otras dos clases $\left(\mathrm{P}_{2}\right.$ y $\left.\mathrm{P}_{3}\right)$ que no fueron diferentes entre ellas (Tabla 8). Ya que estas yuntas de bueyes presentaron productividades similares en las tres clases de pendientes (Tabla 6), esta diferencia probablemente fue provocada por la variable "costo unitario" que presentó valores significativamente diferentes entre las clases de pendientes. A diferencia del skidder J-80A en el cual se registraron productividades significativamente diferentes (decrecientes) a lo largo de todas las pendientes, el John Deere mantuvo desempeños similares en las dos primeras clases de pendiente antes de presentar una caída significativa en la tercera clase (Tabla 8). Esto indica la capacidad de este skidder de mantener su desempeño hasta determinada pendiente (16\% en el presente estudio).

Las yuntas de bueyes y los skidders John Deere 548E, recomendados en este estudio, junto con los skidders LKT81 y los tractores arrastradores TDT-55 A, fueron los principales medios de extracción de madera en las últimas décadas en Cuba. Los skidders John Deere 548E son los principales medios de extracción utilizados en la empresa Macurije en la última década y en los últimos cinco años, los skidders J-80A están siendo probados, principalmente por sus menores costos de adquisición, en comparación al skidder John Deere 548E. Esos modelos de skidders, a pesar de no ser los más actuales, ni los más productivos, atienden las exigencias productivas de la empresa Macurije que procura mejorar su desempeño y/o invertir en maquinaria más económica. Los resultados obtenidos del estudio comparativo de los desempeños los dos principales medios de extracción de madera de esa empresa (skidder John Deere $548 \mathrm{E}$ y yunta de bueyes) con la nueva adquisicion (skidder J$80 \mathrm{~A}$ ) indican mayor rentabilidad de la yunta de bueyes y del skidder John Deere, por lo que se recomenda seguir con esos medios de extracción.

Frente a escenarios conformados por diferentes medios de extracción, sistemas de aprovechamiento (definidos por las características de los camiones disponibles para el transporte) y clases de pendientes generalmente observados en las empresas forestales, se requieren toma de decisiones importantes tales como la adopción del sistema de aprovechamiento adecuado, las asignaciones de los medios de extracción adecuados a cada clase de pendientes en aras de obtener mayores productividades y costos globales minimizados.

Los arreglos factoriales considerados en este estudio (Expresiones 07 y 08) pueden utilizarse para cualquiera de los diseños experimentales generalmente utilizados (diseño completamente al azar y diseño al azar en bloques). A diferencia del arreglo factorial univariado (Expresión 07) generalmente utilizado, el arreglo factorial multivariado (Expresión 08) además de posibilitar el análisis de los 
efectos de factores y sus interacciones en el fenómeno estudiado (extracción de madera), permitió realizar evaluaciones en base a múltiples criterios o variables (análisis multivariable de la varianza). Aunque en este estudio se utilizaron dos criterios (productividad y costo unitario) se puede utilizar la cantidad que se juzgue necesaria para comparaciones más rigurosas en pos de toma de decisiones más acertadas.

\section{CONCLUSIONES}

$\mathrm{El}$ arreglo factorial multivariado es un enfoque viable para una evaluación detallada del desempeño de los medios de extracción de madera;

La edad de los rodales es proporcional a la productividad e inversamente proporcional a los costos unitarios de extracción de madera;

El sistema de aprovechamiento de troncos enteros resultó ser más rentable que el de madera corta;

La pendiente fue directamente proporcional a los costos e inversamente a las productividades de extracción de madera;

El skidder John Deere 548E fue el más económico y productivo de los dos skidders evaluados y, por tanto, es el recomendado para la extracción semimecanizada de madera en la empresa Macurije;

La yunta de bueyes, a pesar de resultar la opción más rentable de todos los medios de extracción evaluados, por su baja productividad, solo podrá ser utilizado en combinación con otros medios de extracción (Ejemplo: John Deere 548E) o en periodos de producción desacelerada. Su uso exclusivo es casi impracticable ya que implicaría aumentar significativamente su cantidad para compensar su baja productividad y por consiguiente satisfacer las demandas continuas de madera en las unidades de transformación primaria de la empresa Macurije.

\section{RECONOCIMIENTOS}

Los autores agradecen: al programa PEC-PG de CAPES/Brasil, a la Empresa Forestal Integral Macurije (Pinar del Río/Cuba), al Programa de Posgrado en Ciencias
Forestales de la Universidad Federal Rural de Pernambuco (PPGCF-UFRPE/Brasil) y al Departamento Forestal de la Universidad de Pinar del Río "Hermanos Saiz Montes de Oca" (DF-UPR/Cuba).

\section{REFERENCIAS}

Barnes, R. M. (1977). Estudos de movimentos e de tempos: projeto e medida do trabalho (6a ed.). São Paulo: E. Blucher.

Behjou, F. K., Majnounian, B., Namiranian, M., \& Dvořák, J. (2008). Time study and skidding capacity of the wheeled skidder Timberjack 450C in Caspian forests. Journal of Forest Science, 54(4), 183-188. doi: 10.17221/5/2008-JFS

Box, G. E. P. (1949). A general distribution theory for a class of likelihood criteria. Biometrika, 36(3-4), 317-346. doi: 10.1093/biomet/36.3-4.317

Bray, J. H. \& Maxwell, S.E. (1985). Multivariate analysis of variance. Sageuniversity paper series on quantitative applications in the social sciences, 07-054. Newbury Park, CA: Sage.

Cándano, F. (2003). Aprovechamiento forestal. Pinar del Rio, Cuba: Editorial Científico Técnica.

Cándano, F. (1998). Propuesta para el perfeccionamiento de la tecnología de aprovechamiento de madera en rodales de Pinus caribaea en la provincia de Pinar del Río. Tesis de doctorado. Universidad de Pinar del Rio, Pinar del Rio, Cuba.

Đuka, A., Pentek, T., Horvat, D., \& Poršinsky, T. (2016). Modelling of Downhill timber skidding: bigger load - bigger slope. Croatian Journal of Forest Engineering, 37(1), 139-150.

Dykstra, D.P. \& Heinrich, R. (1996). FAO Model code of forest harvesting practice. Rome, Italy: FAO.

Elliasson, L. (1999). Simulation of thinning with a single-grip harvester. Forest Science, 45(1), 26-34. doi: 10.1093/forestscience/45.1.26.

Ezzati, S., Najafi, A., \& Durston, T. (2011). Impact of animal logging on soil physical properties in mule trail in Hyrcanian forests. Transportation Research Part D: Transport and Environment, 16(4), 316320. doi: 10.1016/j.trd.2011.01.013

Guera, O. G. M., da Silva, J. A. A., Ferreira, R. L. C., Álvarez L. D., \& Barrero M. H. (2019). Modelos matemáticos para la determinación del turno óptimo en plantaciones forestales. Madera y Bosques, 25(1), e2511636. doi: 10.21829/myb.2019.2511636

Hair Jr, J. F., Anderson, R. E., Tatham, R. L., \& Black, W.C. (2005). Análise multivariada de dados (5a ed.). Porto Alegre: Bookman. 
Hiesl, P. \& Benjamin, J. G. (2013). Applicability of international harvesting equipment productivity studies in Maine, USA: A Literature Review. Forests, 4(4), 898-921. doi: 10.3390/f4040898

Holtzscher, M. A. \& Lanford, B.L. (1997). Tree diameter effects on cost and productivity of cut-to-length systems. Forest Products Journal, Madison, 47(3), 25-30.

Hotelling, H. (1931). The Generalization of Student's Ratio. The Annals of Mathematical Statistics, 2(3), 360-378. doi: 10.1214/aoms/1177732979

Kutner, M. H., Nachtsheim, C. J., Neter, J., \& Li, W. (2005). Applied linear statistical models. McGraw-Hill/Irwin.

Lago, M. J. (2013). Referencia de Procli. Recuperado de http://www.uhu.es/03009/procli/ref_procli.pdf $\geq$

Leite, E. S., Minette, L. J., Fernandes, H.C., Souza, A.P., Amaral, E. J., \& Lacerda, E. G. (2014). Desempenho do harvester na colheita de eucalipto em diferentes espaçamentos e declividades. Revista Árvore, 38(1), 95-102. doi: 10.1590/S0100-67622014000100009

Levene, H. (1960). Robust tests for equality of variances. In: I. Olkin (Ed.), Contributions to probability and statistics: essays in honor of Harold Hotelling (pp. 278-292). Palo Alto Stanford University Press.

Lopes, E.S. Da \& Diniz, C. C. C. (2015). Produtividade do trator florestal chocker skidder na extração de madeira em terrenos declivosos. Floresta, 45(3), 625-634. doi: 10.5380/rf.v45i3.36409

Lopes, E. S., Missel, J. W. P., Dias, A. N., \& Fiedler, N. C. (2007). Avaliação técnica do trator florestal arrastador "skidder" com diferentes tipos de rodados na extração de madeira em povoamentos de pinus. Revista Arvore, 31(6), 1053-1061. doi: 10.1590/S0100-67622007000600010

Lopes, E. S., Sampietro, J. A., Lombardi, K. C., \& Dias, A. N. (2011). Avaliação da umidade na compactação do solo submetido ao tráfego de máquinas de colheita florestal. Revista Árvore, 35(3), 659-667. doi: 10.1590/S0100-67622011000400010

Machado, C. C. (2014). Colheita florestal. Minas Gerais: Editora UFV.

Machado, C.C. \& Lopes, E.S. (2000). Análise da influência do comprimento de toras de eucalipto na produtividade e custo da colheita e transporte florestal. Cerne, 6(2), 124-129.

Mardia, K. V. (1970). Measures of multivariate skewness and kurtosis with applications. Biometrika, 57(3), 519-530. doi: 10.1093/biomet/57.3.519

Maroco, J. (2007). Análise Estatística - Com Utilização do SPSS (3a ed.). Lisboa: Edições Sílabo.

Medel, H. B., Equino, O. P., Lazo, D. A., \& Guera, M. (2011). Determinación del turno de corta para Pinus caribaea var. caribaea en la Empresa Forestal Integral "Macurije". Floresta e Ambiente, 18(1), 109-116. doi: 10.4322/floram.2011.028.

Mousavi, R. (2012). Effect of log length on productivity and cost of Timberjack 450C skidder in the Hyrcanian forest in Iran. Journal of forest science, 58(11), 473- 482. doi: 10.17221/85/2011-JFS

O'rourke, N., Hatcher, L., \& Stepanski, E.J. (2005). Using SAS for Univariate and Multivariate Statistics. Cary, NC: SAS Institute, Inc.

Pereira, A. L. N., Lopes, E. S., \& Dias, A. N. (2015). Análise técnica e de custo do feller buncher skidder na colheita de madeira em diferentes produtividades do povoamento. Ciência Florestal, 25(4), 981-989. doi: $10.5902 / 1980509820659$

Sampietro, J. A. \& Lopes, E. S. (2011). Compactação de um Cambissolo e Neossolo submetidos a diferentes intensidades de tráfego de Feller Buncher e Skidder. Scientia Forestalis, 39(90), 265-272.

Sant'anna, C. M. (1992). Fatores humanos relacionados com a produtividade do operador de motosserra no corte florestal. 1992. Tesis de maestría. Universidade Federal de Viçosa, Viçosa, Brasil.

Santos, L. N., Fernades, H. C., Silva, M. L., Teixeira, M. M., \& Souza, A. P. (2016). Avaliação de custos da operação de extração da madeira com forwarder. Cerne, 22(1), 27-34. doi: 10.1590/01047760201622012076

Santos, S. L. M. \& Machado, C. C. (1995). Análise técnico-econômica do processamento de madeira de eucalipto em áreas planas, utilizando o processador. Revista Árvore, 19(3), 346-357.

SAS Institute Inc. (1999). SAS/STAT user's guide. Version 8 (computer manual).

Schettino, S., Minette, L. J., \& Souza, A. P. (2015). Correlação entre volumetria de florestas de eucalipto e produtividade e custos de máquinas de colheita de madeira. Revista Árvore, 39(5), 935-942. doi: 10.1590/0100-67622015000500016

Seixas, F. \& Castro, G. P. (2014). Extração. En C.C. Machado (Ed.), Colheita Florestal (pp. 106-161). Viçosa: Editora UFV.

Shapiro, S. S. \& Wilk, M. B. (1965). An analysis of variance test for normality (complete samples). Biometrika, 52(3-4), 591-611. doi: 10.1093/biomet/52.3-4.591

Szymczak, D. A., Brun, E. J., Reinert, D. J., Frigotto, T., Mazzalira, C. C., Dal'Col Lúcio, A., \& Marafiga, J. (2014). Compactação do solo causada por tratores florestais na colheita de Pinus taeda L. na região sudoeste do Paraná. Revista Árvore, 38(4), 641-648. doi: 10.1590/S0100-67622014000400007

Trzesniowski, A. (1993). Wozu Walderschliebung in Österreich Österreichische Forstzeitung, 104(7), 5-7. 
Tukey, J. (1949). Comparing individual means in the analysis of variance. Biometrics, 5(2), 99-114. doi: 10.2307/3001913

Manuscrito recibido el 6 de marzo de 2019

Aceptado el 11 de octubre de 2019

Publicado el 24 de abril de 2020
Este documento se debe citar como:

Guera, O. G. M., da Silva, J. A. A., Ferreira, R. L. C., Álvarez L., D. A., Barrero M., H., \& Díaz P., A. L. (2020). Enfoque multivariado en experimentos de extracción de madera en plantaciones forestales. Madera y Bosques, 26(2), e2621934. doi: 10.21829/myb.2020.2621934

Madera y Bosques por Instituto de Ecología, A.C. se distribuye bajo una Licencia Creative Commons Atribución-NoComercialCompartirlgual 4.0 Internacional. 